\title{
HYGROSCOPIC BIOMIMETIC TRANSDUCERS MADE FROM CNT-HYDROGEL COMPOSITES
}

\author{
M. De Volder ${ }^{1}$, S. Tawfick ${ }^{2}$, D. Copic ${ }^{2}$ and A.J. Hart ${ }^{2}$ \\ ${ }^{1}$ IMEC, Heverlee, Belgium and KULeuven, Leuven - Belgium \\ ${ }^{2}$ The University of Michigan, Ann Arbor, MI - USA
}

\begin{abstract}
Plants as well as other biological organisms achieve directed movements by fibres that constraint and direct the isotropic expansion of a matrix material. In order to mimic these actuators, complex arrangements of rigid fibres must be achieved, which is challenging, especially at small scales. In this paper, a new method to organize carbon nanotubes (CNTs) into complex shapes is employed to create a framework for hydrogel infiltration. These CNT frameworks can be realized as iris, needle and bridge architectures, and after hydrogel infiltration, they show directed actuation in response to water uptake. Finally, we show how the latter can be employed as a novel hygroscopic sensor.
\end{abstract}

\section{KEYWORDS}

Microactuator, Microsensor, Carbon Nanotube, CNT, Hydrogel, Biomimetic

\section{INTRODUCTION}

While the fabrication of mechanical actuators typically involves the selection and assembly of discrete components such as actuators and transmissions, natural systems generate motion using smart composite materials that combine force generation and motion transmission capabilities [1]. For instance, motions of plants are typically directed by the lateral expansion of stiff cellulose fibrils embedded in a matrix $[2,3]$. The fibrils convert isotropic swelling into a directed anisotropic actuation such as a bending or twisting motion. Thus, the geometry and organization of nanoscale filaments, and the collective interaction among large numbers of cells enables complex motions in response to simple stimuli such as, humidity, temperature, and light. Examples are found in the opening and closing of pine cones and wheat awns, the orientation of flowers and many other plant movements [1-5].

These evolutionary design principles could greatly benefit the performance of engineered materials in many applications including active composite laminates and microstructured tissue scaffolds [4]. The non-muscular hydraulic movement of plants, has been mimicked in macro-scale devices known as nastic actuators [5-7]. In these actuators, the winding direction of laminated fibers around a tube determines the direction of motion, and the actuators can expand axially or simultaneously contract and twist. Due to the geometrical complexity needed for the fiber winding, current nastic actuators are only fabricated at large scales. However, as in plant cells, the actuation speed is limited by the rate of fluid transport through the system [5], and therefore miniaturisation is greatly improving the response time of these systems. For instance, in plants and fungi, only structures with sub-millimeter dimensions are able to achieve time constants below 1 second [5].

Here, we report the fabrication and simulation of hygrotropic nanocomposite microstructures, which change their shape in response to ambient humidity and therefore mimic the actuation behaviour of plants. The fabrication of these structures is enabled by capillary forming, a new approach to shape aligned carbon nanotubes (CNTs) into complex geometries [8]. These structures are used as a framework which we infiltrate with hydrogels thus forming CNT (fiber) / hydrogel (matrix) composites [9-13]. By taking advantage of the anisotropy of the high aspect ratio CNTs $(>10,000)$ that run along the CNT scaffolds, the resulting microachitectures transform the simple isotropic swelling of the hydrogel in directional movements. This new biomimetic approach complements classic hydraulic microactuators [14].

\section{FABRICATION METHOD}

The active CNT-hydrogel composite microstructures are fabricated as shown in Fig. 1. Patterned microstructures of vertically aligned CNTs ("forests") are grown by chemical vapour deposition (CVD) on silicon wafers using a lithographically-patterned supported catalyst consisting of alumina and iron. While lithographic patterning of the catalyst layer enables precise control of the placement of CNTs on a silicon wafers, CVD "grown" CNT forests are typically limited to $2 \mathrm{D}$ geometries with a low density of CNTs, typically $1-5 \%$ of an ideal hexagonally packed configuration.

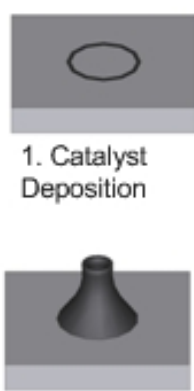

4. Densification

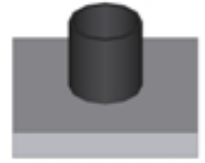

2. CNT Growth

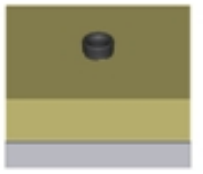

5. Hydrogel

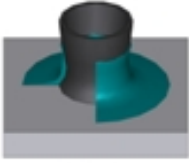

3. Solvent Condensation

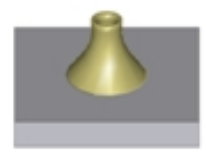

6. Spin-coating
Figure 1: Illustration of the transducer fabrication process. 
These as-grown CNT forests are extremely fragile, and not suitable for the fabrication of bio-mimetic actuators. Therefore, in the following step, the CNTs are capillary formed by forces resulting from the condensation and subsequent evaporation of acetone on the substrate. This capillary densification $[8,15,16]$ process is known to significantly increase the packing density of the CNTs, and therefore increases the collective mechanical stiffness and robustness of the microstructures. Further, we observed that depending on the initial geometry of the CNT structures, the elastocapillary interaction results in different types of stresses which induce a transformation of the initial 2D geometry into a 3D shape. As shown in Figure 1A axisymmetric shapes such as cylinders contract into cones, and also more complex bending and bridges structures will be shown further on [8].

Owing to the robustness of the capillary formed CNTs and the strong capillary interactions between the CNTs and the chosen hydrogel, the composite structures can be formed by spin coating without causing distortions to the original geometry of the CNT templates. The hydrogel used in this study consists of 2-hydroxyethylmethacrylate monomer (HEMA) mixed with a cross-linker ethyleneglycoldimethacrylate (EGDMA) and a photoinitiator. The spin-coating parameters can be chosen to retain the hydrogel exclusively within the CNT structures (Figure 2). Alternatively, by using lower spin-coating speeds, we observed it is possible to locally trap hydrogel droplets within microstructures (Figure $3 b$ ).

\section{MEASUREMENTS AND SIMULATIONS}

Fig. 2 illustrates the basic operation of the actuation principle of CNT-Hydrogel iris.
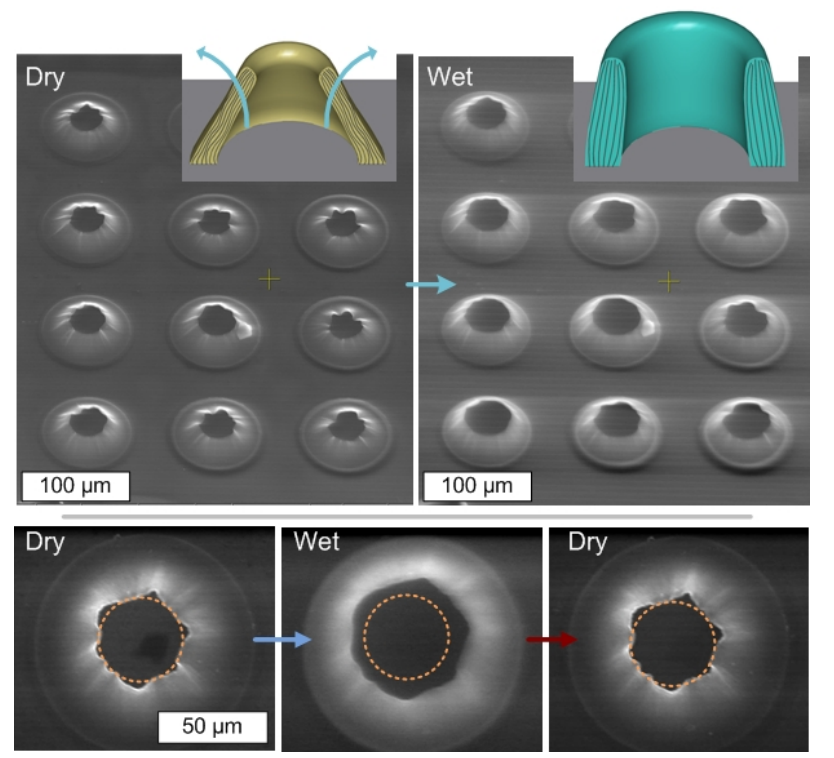

Figure 2: Illustration of CNT-Hydrogel well actuation. Top: Tilted view of an array of wells in dry and wet state. Bottom: Top-view of a well as it expands and contracts.
Figure 2 is obtained by in situ observation of hydrogel using a FEI-Quanta environmental scanning electron microscopy (ESEM). The samples are mounted on a cooling stage $\left(0^{\circ} \mathrm{C}\right)$ and the chamber is evacuated $(0.01$ Torr). Water pressure in the chamber is then slowly increased to 5 Torr and imaging is performed while condensation occurs on the sample. These experiments show that the wells expand upon moisture uptake. For instance, we measured that a well with an initial opening diameter of $140 \mu \mathrm{m}$ increased to $175 \mu \mathrm{m}$ upon wetting. These structures could be used as active irises or active containers.

Alternatively it is also possible to apply this process to bending CNT pillars. Figure 3a shows an arrangement of folded CNT beams in an out of plane cantilever arrangement. The vertical beam bends with an angle of approximately $20^{\circ}$ upon wetting. By reducing the spincoating speed, hydrogel droplets can be caught in the microstructures as shown in Fig. 3B. Upon wetting, the pillars shown in Fig. 3B tilted outward by $5^{\circ}$.
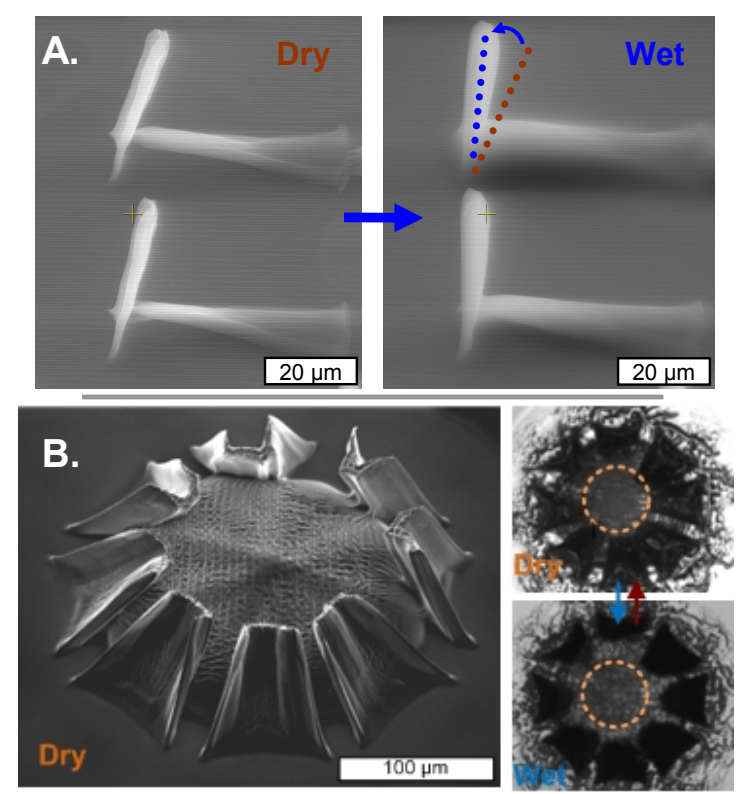

Figure 3: (A) Cantilever arrangement of folded CNT pillars in dry (left) and wet (right) state. (B) Right: Circular arrangement of inward bending CNT-pillars with a hydrogel droplet trapped in the center. Left: Top-view of the structure in dry and wet state.

To further understand the relationship between the geometry of the nanocomposites and their actuation, we fabricated arrays of iris structures with different opening angles, and compared the measured actuation strokes (Diameter in wet condition $\mathrm{D}_{\text {wet }}$ vs. diameter in dry condition $\mathrm{D}_{\text {dry }}$ ) to results from a finite element model (FEM). The initial wall thickness was measured by cutting the structures using a focused ion beam (FIB) (e.g., $\approx 2.5 \mu \mathrm{m}$ in Fig. $4 \mathrm{a}$ ). The dimensions of the structures were then used as inputs to FEM simulations. The actuation was simulated as an anisotropic swelling of the CNT-Hydrogel composite perpendicular to the alignment 
of the CNTs. This model considers the CNT scaffold to be much stiffer in the direction of the CNTs than perpendicular on the alignment.
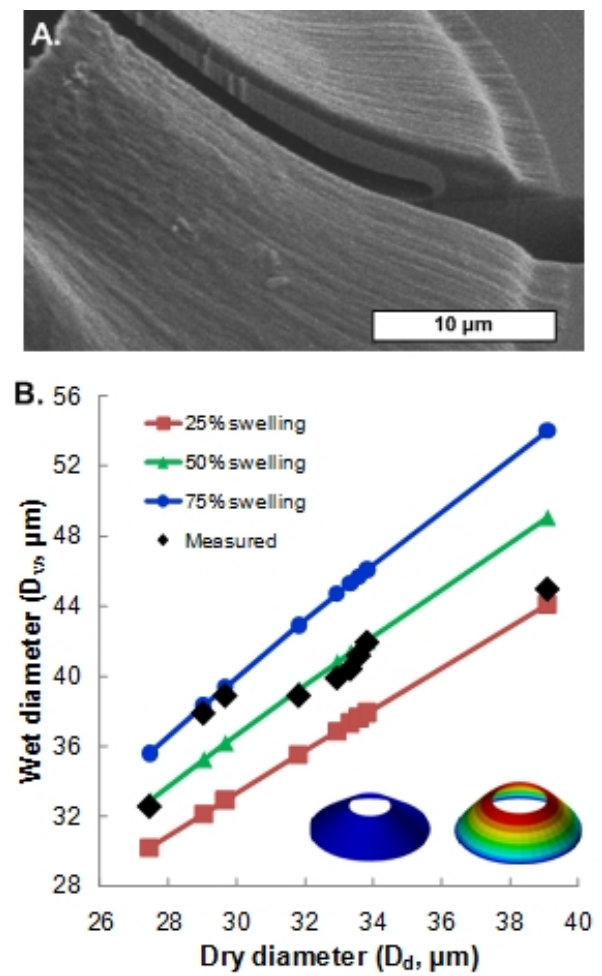

Figure 4: (A) FIB section of a CNT-Hydrogel well in dry state. (B) FEM simulations of well actuation.

Fig. 4b illustrates that the FEM model captures the "breathing" behaviour of the iris, where displacement is largest at the rim. Both measurements and simulations show a linear relationship between $D_{\text {dry }}$ and $D_{\text {wet }}$, and the measured data are bounded by simulation results where the amount of swelling is bound between $25-75 \%$, and is closest to the trend for $50 \%$ swelling. The simulations further predict that the actuation stroke is highly sensitive to the length of the CNTs, but is relatively insensitive to the wall thickness. The ability to simulate the shape transformations suggests that more complex structures can be designed using computational models.

In what follows, we show how CNT-hydrogel composites can also be used to fabricate microsensors. Existing hydrogel based sensors are using optical transmission, fiber bragg gratings, fluorescence intensity, and bending cantilevers to detect the swelling of the hydrogel [17]. However, these sensors often need a complex read-out system. Further, CNTs are usually difficult to integrate electrically in microsystems, and therefore, CNT based microsensors described in literature are fabricated by either dielectrophoresis or piece-wise alignment of sensors around randomly dispersed CNTs. These methods often yield a low throughput or a poor repeatability. In this paper, we introduce a new sensor architecture based on the 3D bridge structures shown in Figure 5. In this approach, a ring of CNT micropillars is grown from semicircles of catalyst deposited on conductive TiN electrodes.
Subsequent capillary forming is used to bend the CNT pillars and form them into a 3D bridge structure (Figure 5). The TiN contacts the base of the CNT pillars and the contact between the tips of the CNT pillars closes the circuit. Compared to classic sensors, the method presented above enables deterministic and parallel fabrication. Further, the sensor delivers directly an electrical signal and can easily be miniaturized, resulting is shorter time constants [5][17]. As shown in Figure 4b, repetitive flooding of the entire sample in DI water and drying in ambient atmosphere caused significant (50\%), and reversible change in the electrical resistance measured across the bridge. The resistance change occurs because the swelling increases the lateral space between the CNTs [12]. Prior to drying, the resistivity of the bridge slightly increases in resistance, which is probably due to the capillary force exerted on the bridge as the water meniscus dries out. Interestingly, these experiments also provide insight in the reaction time of the CNT-hydrogel composites. While hydrogels often swell and especially shrink relatively slowly, Figure $4 \mathrm{~b}$ shows that the CNT-hydrogel bridge reaches full expansion within $1 \mathrm{sec}$. Essential for this fast response is that the 3D suspended geometry of the bridge and the high aspect ratio of the beams promote moisture uptake and evaporation. Further, the time constant of porous materials is typically given by $=r^{2} / D$, where $r$ is a characteristic length and D is a diffusion constant. Due to the square dependency of the time constant with the dimensions, scaling down of the CNT scaffolds will further improve reaction times.
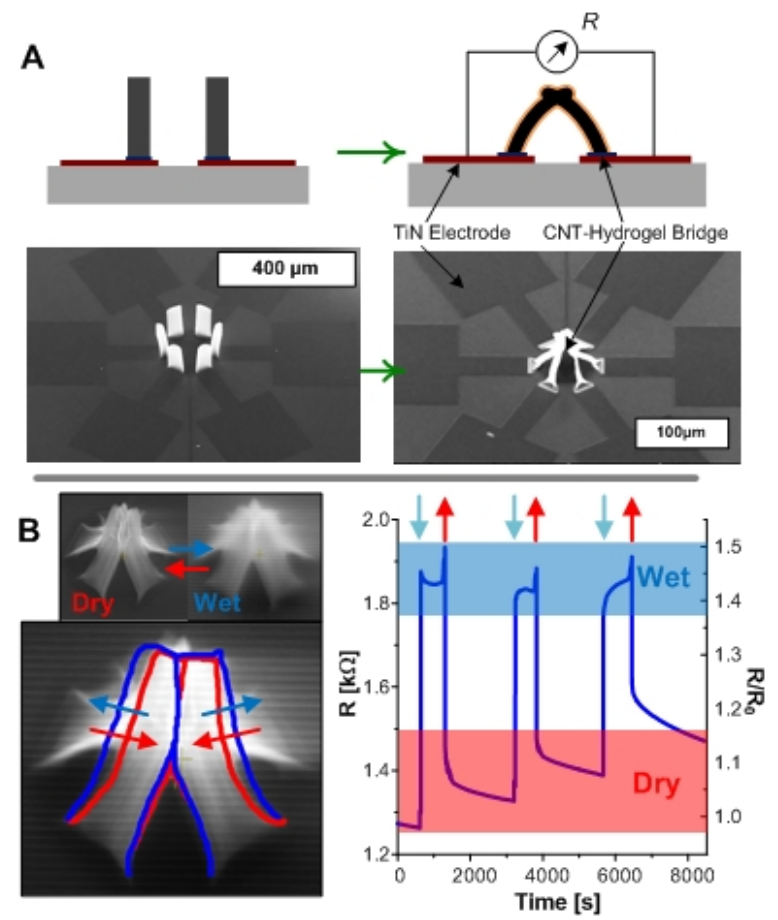

Figure 5: (A) Schematics of hygroscopic sensor based on the CNT-hydrogel composite bridge. (B) SEM images of the sensing bridge in wet and dry state and the corresponding changes in resistance. 
We did not perform extended life time experiments, however we did not observe any deterioration in the actuation over 10 wetting and drying cycles, and after 1 year the samples were re-tested and still showed the same transducer behaviour.

\section{CONCLUSIONS AND OUTLOOK}

Despite the efforts made the past decade to elaborate bio-inspired materials, and gain insight in their morphology, many mechanisms still remain to be elucidated. In this paper, we mimic the nastic actuation of plants by exploiting a new method for fabricating complex CNT-hydrogel composites. Upon moisture uptake these structures deform and this behaviour has been simulated and applied in sensors. By engineering their molecular structure, polymer gels can be made responsive to other stimuli such as light, $\mathrm{pH}$, temperature, biochemical processes, electric and magnetic fields, and we therefore envision that the hydrogel based sensors and actuators introduced in this paper can be tuned to a variety of applications. Since the transduction principle is an inherent material property, we expect these structures retain their active behaviour over long cycle times and long lifetime, promoting their use in remote and inaccessible locations.

\section{ACKNOWLEDGMENTS}

This research was supported by the Belgium Fund for Scientific Research - Flanders (FWO), Belgium, the Nanomanufacturing program of the National Science Foundation (CMMI-0927634), and startup funds to A.J.H. at the University of Michigan.

\section{CONTACT}

M. De Volder: Michael.DeVolder@IMEC.be

A.J. Hart: ajohnh@umich.edu

\section{REFERENCES}

1. Fratzl, P. and F.G. Barth, Biomaterial systems for mechanosensing and actuation. Nature, 2009. 462(7272): p. 442-448.

2. Burgert, I. and P. Fratzl, Actuation systems in plants as prototypes for bioinspired devices. Philosophical Transactions of the Royal Society a-Mathematical Physical and Engineering Sciences, 2009. 367(1893): p. 1541-1557.

3. Burgert, I. and P. Fratzl, Plants control the properties and actuation of their organs through the orientation of cellulose fibrils in their cell walls. Integrative and Comparative Biology, 2009. 49(1): p. $69-79$

4. Sanchez, C., H. Arribart, and M.M.G. Guille, Biomimetism and bioinspiration as tools for the design of innovative materials and systems. Nature Materials, 2005. 4(4): p. 277-288.

5. Skotheim, J.M. and L. Mahadevan, Physical limits and design principles for plant and fungal movements. Science, 2005. 308(5726): p. 1308-1310.
6. Freeman, E. and L.M. Weiland, High Energy Density Nastic Materials: Parameters for Tailoring Active Response. Journal of Intelligent Material Systems and Structures, 2009. 20(2): p. 233-243.

7. Philen, M.K., Y. Shan, P. Prakash, K.W. Wang, C.D. Rahn, A.L. Zydney, and C.E. Bakis, Fibrillar network adaptive structure with ion-transport actuation. Journal of Intelligent Material Systems and Structures, 2007. 18(4): p. 323-334.

8. De Volder, M., S.H. Tawfick, S.J. Park, D. Copic, Z.Z. Zhao, W. Lu, and A.J. Hart, Diverse 3D Microarchitectures Made by Capillary Forming of Carbon Nanotubes. Advanced Materials. 22(39): p. 4384-+.

9. Barone, P.W., H. Yoon, R. Ortiz-Garcia, J.Q. Zhang, J.H. Ahn, J.H. Kim, and M.S. Strano, Modulation of Single-Walled Carbon Nanotube Photoluminescence by Hydrogel Swelling. Acs Nano, 2009. 3(12): p. 3869-3877.

10. Haider, S., S.Y. Park, K. Saeed, and B.L. Farmer, Swelling and electroresponsive characteristics of gelatin immobilized onto multi-walled carbon nanotubes. Sensors and Actuators B-Chemical, 2007. 124(2): p. 517-528.

11. Ogoshi, T., Y. Takashima, H. Yamaguchi, and A. Harada, Chemically-responsive sol-gel transition of supramolecular single-walled carbon nanotubes (SWNTs) hydrogel made by hybrids of SWNTs and cyclodextrins. Journal of the American Chemical Society, 2007. 129(16): p. 4878-+.

12. Yang, Z.H., Z. Cao, H. Sun, and Y. Li, Composite films based on aligned carbon nanotube arrays and a poly(N-isopropyl acrylamide) hydrogel. Advanced Materials, 2008. 20(11): p. 2201-+.

13. Vourdas, N., D. Kontziampasis, G. Kokkoris, V. Constantoudis, A. Goodyear, A. Tserepi, M. Cooke, and E. Gogolides, Plasma directed assembly and organization: bottom-up nanopatterning using top-down technology. Nanotechnology. 21(8).

14. De Volder, M. and D. Reynaerts, Pneumatic and hydraulic microactuators: a review. Journal of Micromechanics and Microengineering. 20(4).

15. Chakrapani, N., B.Q. Wei, A. Carrillo, P.M. Ajayan, and R.S. Kane, Capillarity-driven assembly of two-dimensional cellular carbon nanotube foams. Proceedings of the National Academy of Sciences of the United States of America, 2004. 101(12): p. 4009-4012.

16. Liu, H., S.H. Li, J. Zhai, H.J. Li, Q.S. Zheng, L. Jiang, and D.B. Zhu, Self-assembly of large-scale micropatterns on aligned carbon nanotube films. Angewandte Chemie-International Edition, 2004. 43(9): p. 1146-1149.

17. Richter, A., G. Paschew, S. Klatt, J. Lienig, K.F. Arndt, and H.J.P. Adler, Review on hydrogel-based $\mathrm{pH}$ sensors and microsensors. Sensors, 2008. 8(1): p. 561-581. 